\title{
Study on acoustic analysis of Cleveland Dam waterfall sound
}

\author{
Zhixing Tian', Bong-Young Kim² and Myung-Jin Bae ${ }^{3 *}$ \\ ${ }^{1}$ Soong-sil University, Department of Information and telecommunication Engineering, Seoul, 06978, Korea \\ Orcid Id : 0000-0003-3882-2459 \\ ${ }^{2}$ Soong-sil University, Department of Information and telecommunication Engineering, Seoul, 06978, Korea \\ Orcid Id : 0000-0002-3553-039X \\ ${ }^{3}$ Soong-sil University, Department of Information and telecommunication Engineering, Seoul, 06978, Korea \\ Orcid Id : 0000-0002-7585-0400
}

\begin{abstract}
:
Sound therapy has been used as a feasible method of psychotherapy in psychology for many years and has many mature research results [1]. Natural sounds such as rain, sea waves, wind, waterfalls, etc. are also widely regarded as healthy sounds that relieve stress and relax the mind and body. This provides a good way for people who are living in a fastpaced life and who have been troubled by urban noise for a long time to relieve stress and maintain health. In fact, different acoustic characteristics of sound will bring different psychological effects. Cleveland Dam waterfall sound is a healthy artificial waterfall sound. This paper uses spectrum to analyze its acoustic spectrum characteristics, which is related to the structure of the dam and the resonance of water sound with the surrounding environment. Analyze his effect through brain wave measurement experiment. Because the sound energy of Cleveland Dam waterfall induces many $\alpha$ waves and suppresses $\delta$ waves, it is proved to be a healthy sound with a sense of comfort and relaxation.
\end{abstract}

Keyword: Cleveland Dam, acoustic, waterfall sound, brain wave, frequency spectrum

\section{INTRODUCTION}

People in modern cities are more and more concerned about their health. They have many ways to keep their healthy, but they rarely pay attention to the effect of their hearing on their own health. In fact, the sound is closely related to people's health. The human auditory system is an extremely sensitive organ of alertness, and it remains in working condition even when sleeping. It is a sensor that people perceive changes in the surrounding environment. Therefore, the auditory system is connected to the auditory nervous system of the brain through numerous nerves, and sound affects the nerve regulation and hormone regulation in the body, which is ultimately reflected in the human reaction and emotion. Studies have shown that sound can have a specific psychological effect on people and can change people's emotions. So, there are many people who listen to music to relieve stress [2]. On the contrary, urban traffic noise is an unpleasant sound. People who work in such a noisy environment for a long time will feel irritable, anxious, and then tired, and reduce their cognitive ability and mobility, affecting work efficiency. In life, urban traffic noise can affect people's sleep quality. This is the negative effect of sound [3].

However, the sounds of wind, rain, running water, waterfalls, and waves etc. are all white noise and pink noise in the natural environment. They are all comfortable and relaxing sounds. Being in such an acoustic environment can make people feel relaxed and beneficial to our health [4]. This paper studies the sound of a man-made dam waterfall in Canada. The Cleveland Dam is a 91-meter-high concrete dam built in 1954, located on the upper reaches of the Capilano River in North Vancouver, British Columbia. It blocks Lake Capilano, which provides fresh drinking water. When the sluice gate of the spillway is opened, the scene where the water flows from the lake to the bottom of the canyon more than 90 meters deep is particularly spectacular. Like a huge waterfall. The loud waterfall sound can be clearly heard within a radius of $1 \mathrm{~km}$. Figure 1 is a top view of Cleveland Dam waterfall sound [5] [6].

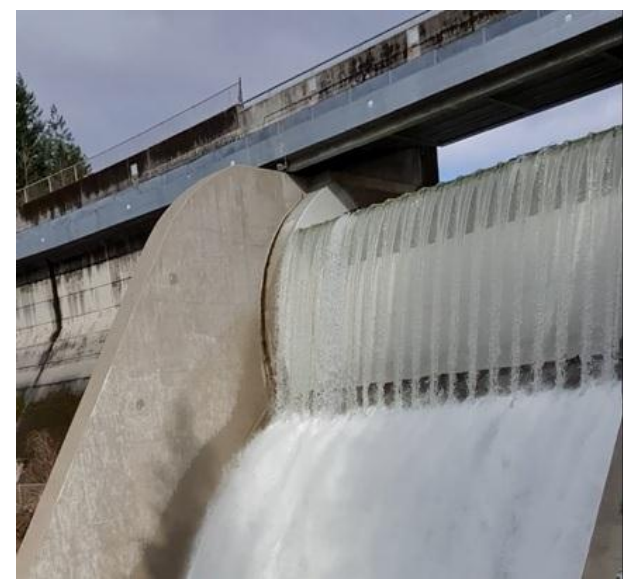

Figure 1. Cleveland Dam

This paper is about the acoustics of the sound of a man-made dam waterfall in Canada and how it affects people's health. Therefore, it mainly conducted by spectrum analysis and brain wave measurement of Cleveland Dam waterfall sound. Combined with the Sori Sound Engineering laboratory's previous analysis of Sunwapta Fall's acoustic analysis of natural waterfalls. In chapter 2 , introduced the characteristics and applications of white noise. In chapter 3 , performed power 
spectrum analysis and energy spectrum analysis on it. In chapter 4 , introduced brain waves. In chapter 5 , conducted brain wave measurement experiments on the subjects under three sound environments and analyzed it and get the experimental result. In chapter 6 , the conclusion.

\section{WHITE NOISE}

White noise refers to noise whose power spectral density is constant over the entire frequency domain. Random noise with the same energy density at all frequencies is called white noise. Acoustically, white noise has an even energy distribution in all frequency bands within the audible range of the human ear (20 to $20000 \mathrm{~Hz}$ ) [7]. With flat spectrum characteristics. This kind of sound is widespread in the natural environment, such as the sound of rain, the sound of sea waves, the sound of wind blowing leaves, the sound of waterfalls, etc.

Because natural white noise has a wide spectrum and flat power spectral density in the audible range of the human ear. Therefore, natural white noise is usually regarded as a natural comfortable and relaxing sound. In psychology, listening to white noise can induce $\alpha$ brain waves, which can make people relax. Because it exists widely in nature, people can easily obtain it. Many people reduce stress and relax by listening directly to the sound of natural wind, waterfalls, and waves. In medicine, white noise has been used as a treatment method. It has the effect of treating tinnitus and helping sleep. It can even treat mild depression and mental concentration disorder. Because its energy in the whole frequency band is the same, it can shield the noise accumulated by other energy. This principle is also widely used in our lives. For example, in the movie, the protagonist turns on the shower head in the bathroom, and then chats or calls to prevent eavesdropping. Placing a white noise generator screen in the office can shield the construction noise around the site and so on [8] [9]

\section{SPECTROGRAM AND FREQUENCY SPECTRUM}

Analysis of the Sunwapta Fall spectrogram reveals that Sunwapta has a very flat energy distribution over the entire spectrum. High energy distribution only appears in $100 \sim 500 \mathrm{hz}$ and $6 \sim 9 \mathrm{Khz}$. According to the spectrogram, it is found that the energy distribution of Cleveland Dam waterfall sound is very different from Sunwapta Fall. Its high energy is mainly concentrated in the low-frequency and mid-frequency range of $100 \sim 1 \mathrm{Khz}$. After 3Khz, the energy shows a small amount of uniform distribution on the frequency spectrum. This means that Cleveland Dam waterfall has higher low frequency energy evenly distributed, and it approximates the spectrum of white noise, so it also has the characteristics of white noise. Sunwapta Fall also shows a flat distribution of energy in the spectrum.
[10]. Figure 2 is Waveform and spectrogram of Cleveland Dam waterfall. Figure 2 is the Waveform and spectrogram of Sunwapta Fall.

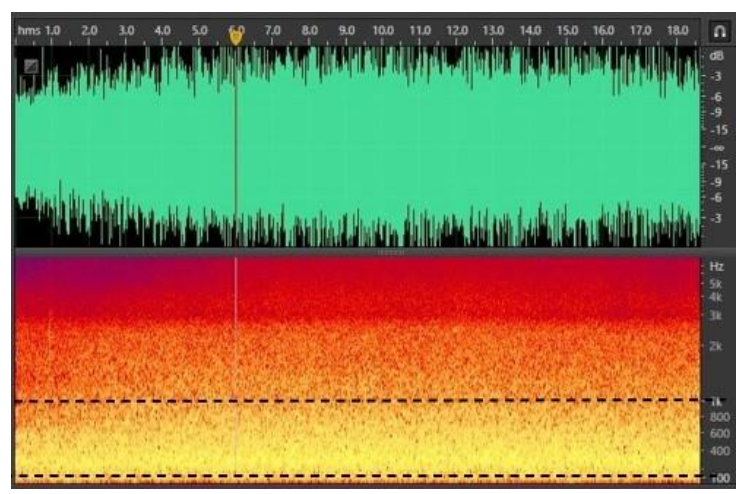

Figure 2. Waveform and spectrogram of Cleveland Dam waterfall

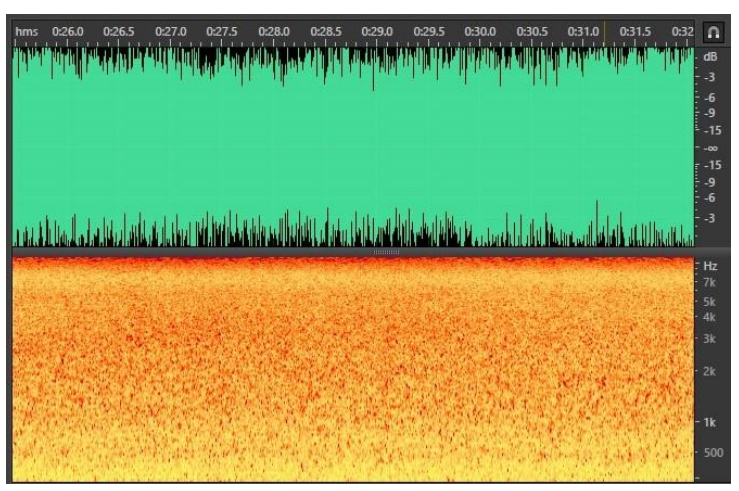

Figure 3. Waveform and spectrogram of Sunwapta Fall

Figure 4 is the spectrum of Sunwapta Fall and Cleveland Dam waterfall. By analyzing it, more spectrum information can be obtained. The energy of Cleveland Dam waterfall sound below $100 \mathrm{hz}$ is very low, and the peak energy to $300 \mathrm{hz}$ increases by more than $35 \mathrm{~dB}$. Then the energy drops sharply in the range of $300 \mathrm{hz}$ to $1 \mathrm{Khz}$. After $1 \mathrm{khz}$ the decline began to slow down to $3 \mathrm{Khz}$. Therefore, a resonant peak appears in the range of $20 \mathrm{hz}-$ $3 \mathrm{Khz}$. It is formed by the resonance of the sound of waterfall and the surrounding canyon terrain. The spectrum energy distribution in the range from $100 \mathrm{hz}-3 \mathrm{kz}$ approximate to the equal loudness curve of human senses. This means that even if the energy varies greatly in this spectrum range, people feel that the sound of each frequency component is the same loudness. People don't get irritated by the excessive energy in a certain frequency band, because humans are more comfortable with equal-loud sounds. After $3 \mathrm{Khz}$, the energy of each frequency is

However, a formant appeared in the high frequency band, and Cleveland Dam waterfall did not. [11] [12]. 


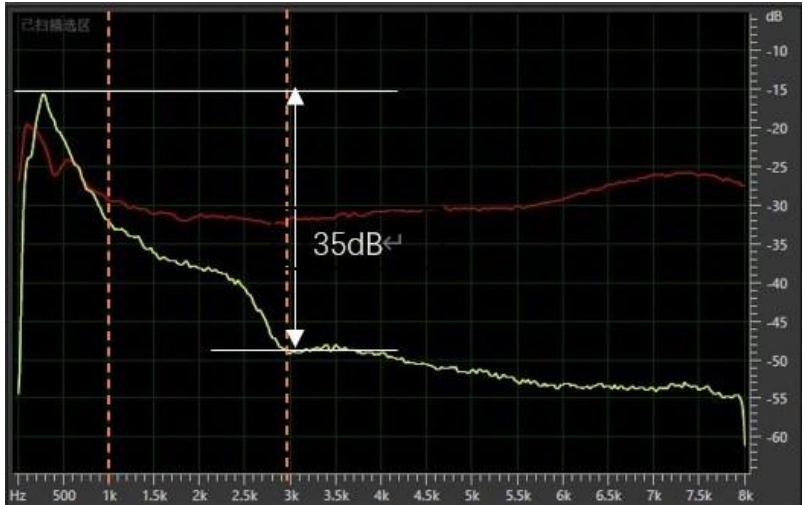

Figure 2. Spectrum analysis of Sunwapta waterfall sound

\section{DEFINITION OF BRAIN WAVES}

possible roles of brain wave include feature binding, information transfer mechanisms and the generation of rhythmic motor output. It is usually converted into EEG signals by EEG measurement. EEG signals can reflect the activity patterns of cerebral cortical neurons. Observation and inspection of EEG is an important method to obtain information on brain activity. according to the frequency and amplitude characteristics of EEG signals, it is mainly divided into four types of EEG waves: $\delta$ wave, $\theta$ wave, $\alpha$ wave, and $\beta$ wave [13]. Beta waves are generally divided into low beta waves $(\beta \mathrm{L})$ and high beta waves $(\beta \mathrm{H})$. The appearance of different brain waves reflects the different brain functional activities of the human brain at this time. Table 1 is the classification of brain waves and brain function [14].

Table 1. Classification of brain waves [14]

\begin{tabular}{|c|c|c|}
\hline $\begin{array}{c}\text { Types } \\
\text { (Frequency) }\end{array}$ & Amplitude & Human brain activity \\
\hline $\begin{array}{l}\delta \text { wave } \\
(1-3 h z)\end{array}$ & $20-200 \mu \mathrm{V}$ & $\begin{array}{l}\text { In most cases, it occurs in deep sleep. Neurons in the cortex are in a } \\
\text { state of simultaneous relaxation, which is an unconscious brain } \\
\text { wave. }\end{array}$ \\
\hline $\begin{array}{l}\theta \text { wave } \\
(4-7 h z)\end{array}$ & $100-150 \mu \mathrm{V}$ & $\begin{array}{l}\text { It is a brain wave that appears between awake and sleep states, it is } \\
\text { the performance of the central nervous system's inhibition state, and } \\
\text { is related to the learning and memory processes. }\end{array}$ \\
\hline $\begin{array}{c}\text { a wave } \\
(8-13 h z)\end{array}$ & $20-100 \mu \mathrm{V}$ & $\begin{array}{l}\text { It is the main brain wave that appears in a normal quiet state, } \\
\text { indicating that in a quiet state, the cerebral cortical nerve cells are in } \\
\text { a state of relay ation and nrenaration for artivity. The appearance of } \\
\alpha \text { Wave can make people feel relaxed quickly. }\end{array}$ \\
\hline $\begin{array}{l}\beta \text { wave } \\
(14-30)\end{array}$ & $5-20 \mu \mathrm{V}$ & $\begin{array}{l}\text { It occurs when people perform behavioral actions and increase } \\
\text { attention, such as thinking about problems or performing } \\
\text { intellectual activities. However, the continuous emergence of a large } \\
\text { number of } \beta \text { waves will give a lot of pressure and tension. }\end{array}$ \\
\hline
\end{tabular}

\section{EXPERIMENTS AND RESULTS}

The brain wave measurement experiment is to measure the brain waves in the three states of two types waterfall sounds and quiet state. In order to ensure the accuracy of the experiment, 4 people aged from 20 to 26 participated in the experiment. First, Sori Sound Engineering Lab has obtained Sound recording resources of Cleveland Dam waterfall and Sunwapta Fall for one minute, with a sampling rate of $16 \mathrm{Khz}$ in wav format recording files. The measuring instrument is a two-channel brain wave measuring instrument produced by Neuro Harmory S, with a bandwidth of $45 \mathrm{~Hz}$ and a sampling rate of $512 \mathrm{~Hz}$. During the measurement, all testees remain still and close their eyes.

First, measure brain waves in a quiet state for three minutes.
Then, after 5 minutes of rest, play the sound of Cleveland Dam waterfall for three minutes, and measure the brain waves. After continuing the rest for 5 minutes, measure the brain waves when listening to Sunwapta Fall. Four testers repeated the above operations.

Figure 5 is a bar chart of the percentage of brain waves before and after the four testees listened to Cleveland Dam waterfall. As shown in the figure, the four testees had the same trend in the percentage of brain waves listening to Cleveland Dam waterfall compared to when they were quiet. Which is the percentage of $\alpha$ waves occupied by the four test subjects increased, the percentage of $\delta$ waves and beta waves decreased, and there was no significant change in theta waves [15]. 
International Journal of Engineering Research and Technology. ISSN 0974-3154, Volume 13, Number 6 (2020), pp. 1159-1164

(C) International Research Publication House. https://dx.doi.org/10.37624/IJERT/13.6.2020.1159-1164

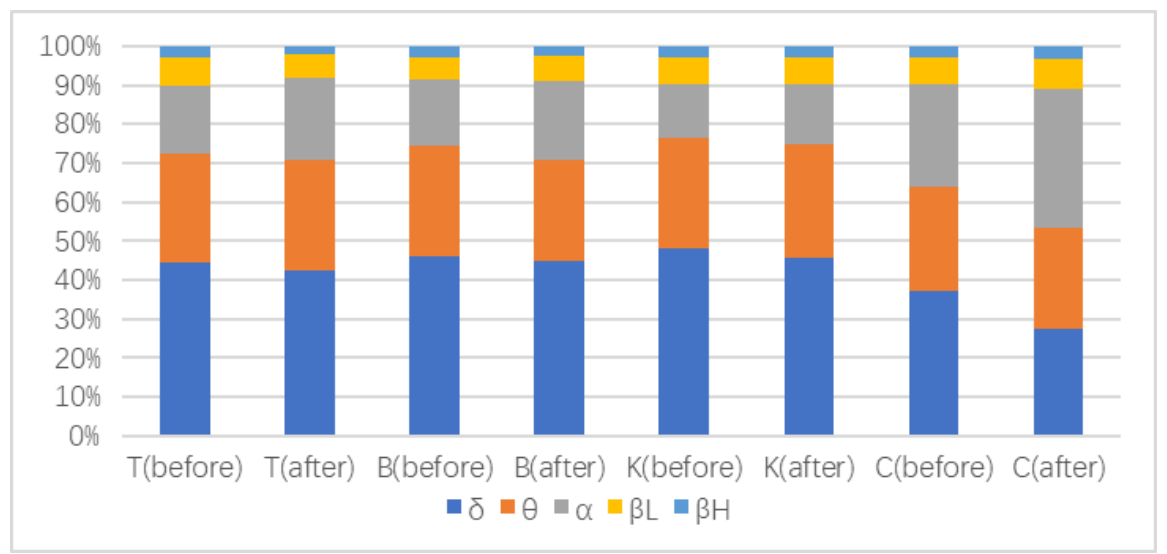

Figure 5. The percentage of brain waves before and after the four testees listened to Cleveland Dam waterfall

Compare and analyze the brain waves of people A in three situations. The following are the EEG Isopotential Mapping of People $\mathrm{A}$ in three states. In a quiet environment, the relative power values of various brain waves are very low, and people $A$ are in a state of no thoughts, depressed mood, and poor cognition and mobility. Conversely, when he listening to the sound of waterfalls, both waterfall sounds induced a lot of brain waves. This is shown in figure 9. However, more alpha waves and beta waves can improve his concentration and mobility, also keep him in a quiet and sober state.

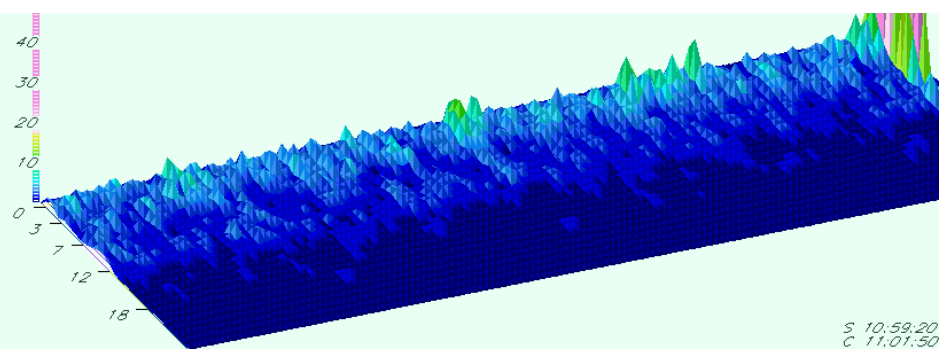

Figure 6. EEG Isopotential Mapping of people A in quiet environment

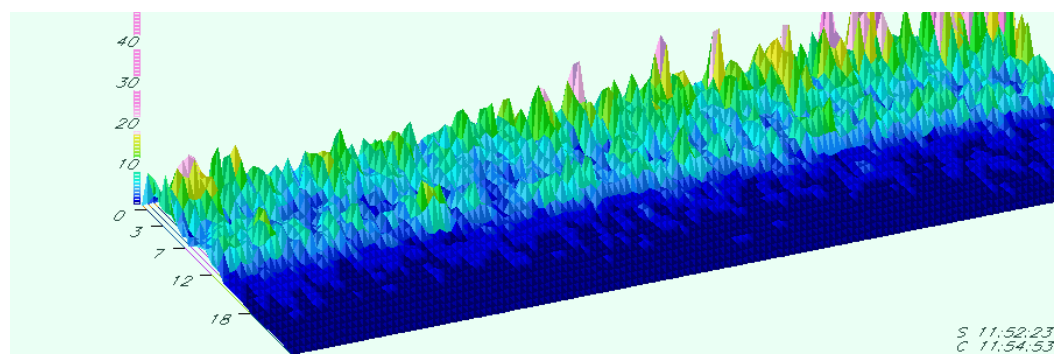

Figure 7. EEG Isopotential Mapping of people A when listening to Cleveland Dam waterfall

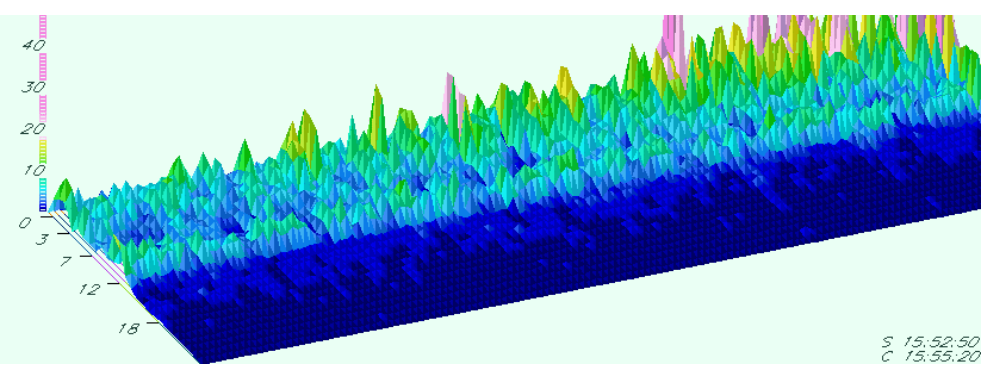

Figure 8. EEG Isopotential Mapping of people A when listening to Sunwapta waterfall 
International Journal of Engineering Research and Technology. ISSN 0974-3154, Volume 13, Number 6 (2020), pp. 1159-1164

(C) International Research Publication House. https://dx.doi.org/10.37624/IJERT/13.6.2020.1159-1164

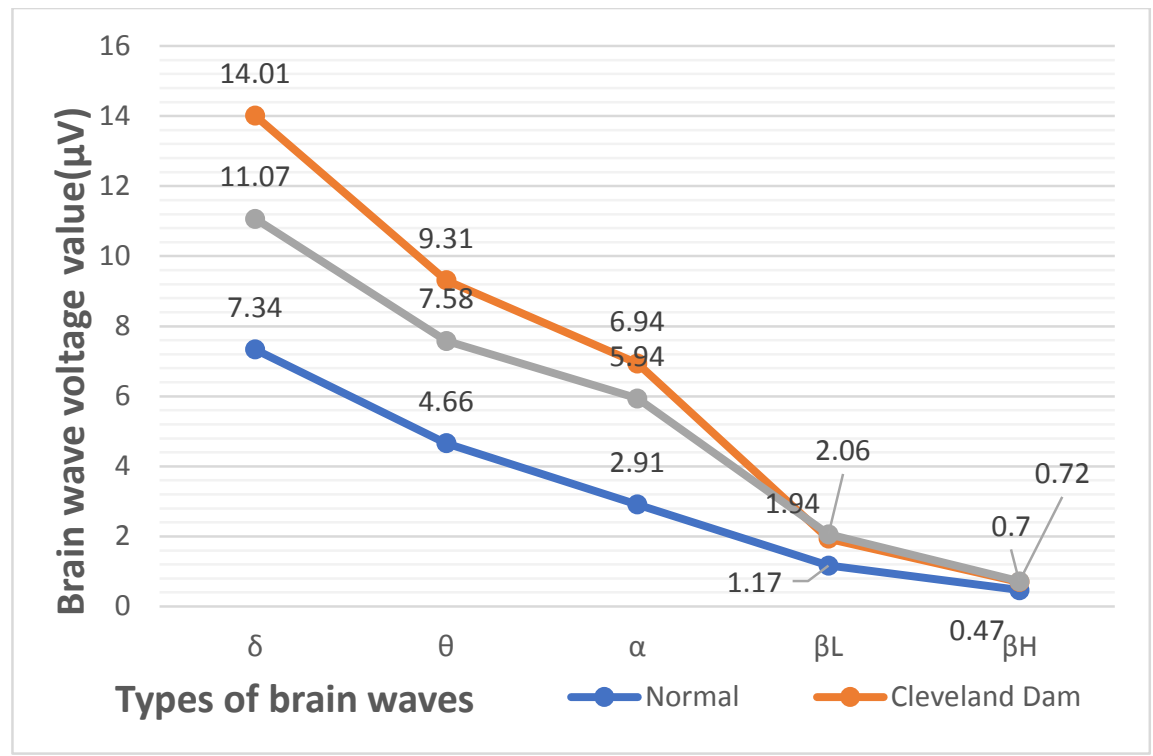

Figure 9. Comparison of various brain waves in three cases

Figure 10 shows the percentage of brain waves in the brain in three cases. Which is reflecting the difference of the working state of the brain under the three cases. The percentage of $\alpha$ waves occupied by the two waterfall sounds has increased significantly, while the proportion of $\delta$ waves has decreased. The $\Theta$ wave has not changed. In the case of $\beta \mathrm{L}$ waves and $\beta \mathrm{H}$ waves, the percentage of $\beta \mathrm{L}$ waves and $\beta \mathrm{H}$ waves in Cleveland Dam waterfall decreases. But Sunwapta Fall has more beta waves than when it is quiet. Therefore, compared with
Sunwapta Fall, Cleveland Dam waterfall has more $\delta$ waves and less $\beta$ waves. In other words, listening to Sunwapta Fall is more conducive to people thinking quietly. listening to Cleveland Dam waterfall has a better relaxation effect, can better enjoy the relaxation and immerse in it. If people can listen to the sound of Cleveland Dam waterfall, hiking in the forest of Capilano Park is a very pleasant thing, it can make people better enjoy relaxation and immerse in the beautiful surroundings [16] [17].

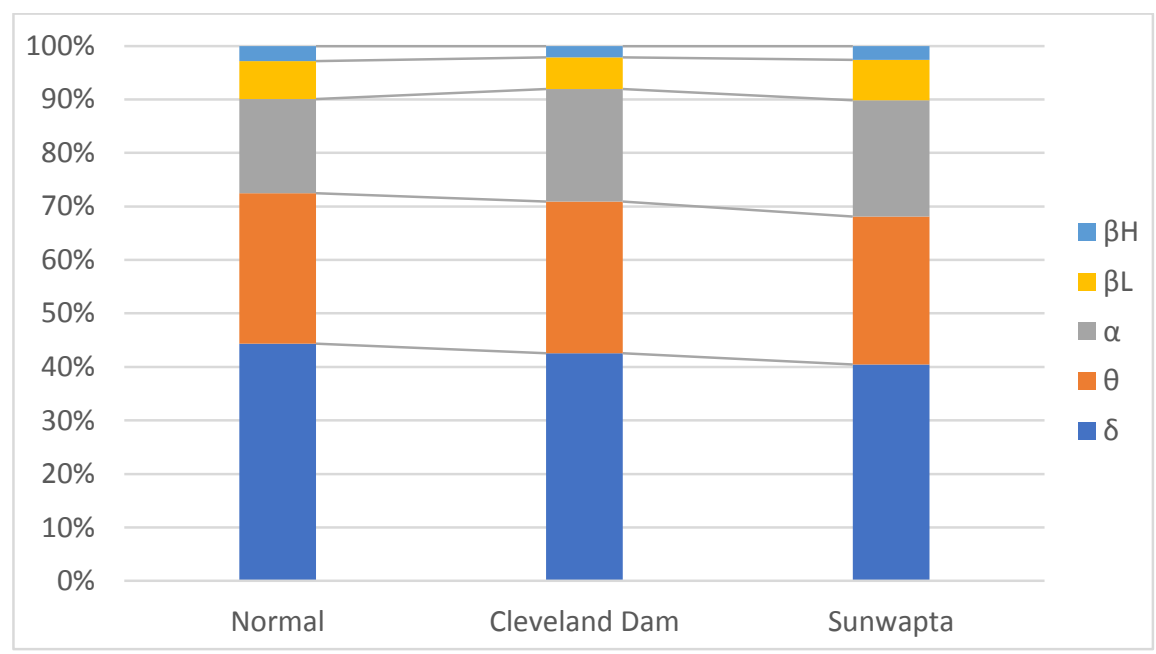

Figure 10. Comparison of the percentage of brain waves in the brain in three cases

\section{CONCLUSION}

Sound is closely related to people's psychological. Thus, the sound can affect people's health. Natural white noise is a kind of sound that is beneficial to people's health. It includes natural wind, rain, waterfall, and waves. They are all sounds that make you feel comfortable and relaxed. However, the acoustic properties of various natural white noises are not identical [18].

Cleveland Dam waterfall is not a naturally formed waterfall, 
and its acoustic characteristics are related to the structure of the dam and the surrounding geographical environment. Compared with Sunwapta natural waterfall sound, it has higher mid-low frequency energy, and high frequency energy is very low. Below $3 \mathrm{khz}$, its spectrum curve is like the equal loudness curve of humans, while above $3 \mathrm{khz}$ is like white noise. In brain wave measurement, it was found that Cleveland Dam waterfall can stimulate $\alpha$ brain wave, reduce $\delta$ brain wave, and no change in $\theta$ wave and $\beta$ wave. This means that more $\delta$ waves are converted into $\alpha$ waves. People will feel that their sleepiness has gradually disappeared, and their ability to perceive the outside world has been strengthened to keep people quiet and relaxed. This is slightly different from the sound of Sunwapta natural waterfall. Sunwapta natural waterfall sound is more conducive to people thinking quietly, while Cleveland Dam waterfall sound can get more relaxation and comfort.

So, whether people listen to the Cleveland Dam waterfall recording after a busy work or listen to it while strolling in the forest of Capilano Park in person, it can help people relieve stress. Listening more to this voice can benefit people's health.

\section{REFERENCE}

[1] Domantė Kučikienè, Rūta Praninskienè. "The impact of music on the bioelectrical oscillations of the brain" Acta Med Litu. 2018; 25(2): 101-106.

[2] Boyd-Brewer, C., McCaffrey, R. "Vibroacoustic sound therapy improves pain management and more". Holistic Nursing Practice Volume 18, Issue 3, May-June 2004, Pages 111-118.

[3] Seonggeon Bae, Myungjin Bae. "A study on the effects of depression using the weighting white noise". Acoustical Society of America, Volume 139, Issue 4.

[4] Ik-Soo Ahn, Bong-young kim, Myung-jin Bae. "A study on the human sensation of the reed wind sound in ASMR". International Journal of Engineering Research and Technology Volume 12, Issue 9, 2019, Pages 14941499.

[5] ancouver's North Shore. "CLEVELAND DAM". [https://vancouversnorthshore.com/attractions/cleveland$\mathrm{dam} /]$.

[6] Wikipedia. "cleveland dam". [https://en.wikipedia.org/wiki/Cleveland_Dam].

[7] Söderlund, Göran; Sverker Sikström; Andrew Smart (2007). "Listen to the noise: Noise is beneficial for cognitive performance in ADHD". Journal of Child Psychology and Psychiatry. 48 (8): 840-847.

[8] Rausch, V. H. (2014). White noise improves learning by modulating activity in dopaminergic midbrain regions and right superior temporal sulcus. Journal of cognitive neuroscience, 1469-1480.

[9] Kyoung-su Yeo, Myungsook Kim, Myung-Jin Bae. "Acoustic characteristics of the forest sounds inducing sleep. Information (Japan) Volume 18, Issue 10, October 2015, Pages 4407-4412.

[10] Zhixing Tian, Bong-Young Kim, Myung-Jin Bae. "Study on the acoustic characteristics of Sunwapta Falls". International Journal of Engineering Research and Technology 13(5), To be published.

[11] Ik-Soo Ahn, Myung-Jin Bae and Seong-Geon Bae. "A comparative analysis of the sound and replay of Asian falls". International Journal of Engineering Research and Technology Volume 12, Issue 11, 2019, Pages 20482051.

[12] Ukjin Song, Eun-young Yi, Myung-Jin Bae. "A study on sound transfer characteristics of the cave concert. Information (Japan) Volume 20, Issue 6, June 2017, Pages 4101-4108.

[13] Nikulin VV, Linkenkaer-Hansen K, Nolte G, Lemm S, Muller KR, Ilmoniemi RJ, Curio G (2007). "A novel mechanism for evoked responses in the human brain". Eur J Neurosci. 25 (10): 3146-3154.

[14] Personal library. "The four brain waves of man". [http://www.360doc.com/content/15/0714/17/15991787_ 484900161.shtml].

[15] Kyoung-su Yeo, Myungsook Kim, Myung-Jin Bae. Acoustic characteristic of music inducing sadness. Information (Japan) Volume 19, Issue 5, May 2016, Pages 1349-1354.

[16] Ik-Soo Ahn, Bong-Young Kim, Kwang-Bock You, Myung-Jin Bae "A Study on the Characteristics of an EEG Based on a Singing Bowl's Sound Frequency", Studies in Computational Intelligence Volume 789, 2019, Pages 233-243.

[17] Mäkinen V, Tiitinen H, May P (2005). "Auditory eventrelated responses are generated independently of ongoing brain activity". NeuroImage. 24 (4): 961-968.

[18] Loewen, Laura J.; Peter Suedfeld (1992-05-01). "Cognitive and Arousal Effects of Masking Office Noise". Environment and Behavior. 24 (3): 381-395. 